\title{
ELM-Based Indonesia Vehicle License Plate Recognition System
}

\author{
Basuki Rahmat ${ }^{1 *}$, Endra Joelianto ${ }^{2}$, I Ketut Eddy Purnama $^{3}$, Mauridhi Hery Purnomo ${ }^{3}$ \\ ${ }^{1}$ Universitas Pembangunan Nasional "Veteran" Jawa Timur, Surabaya, Indonesia \\ ${ }^{2}$ Instrumentation and Control Research Group, Faculty of Industrial Technology, Institut Teknologi Bandung, Bandung 40132, \\ Indonesia \\ ${ }^{3}$ Department of Electrical Engineering, Faculty of Industrial Technology, Institut Teknologi Sepuluh Nopember, Surabaya 60111, \\ Indonesia
}

\begin{abstract}
In this paper, a widely developed learning machine algorithm called Extreme Learning Machine (ELM) is used to recognize Indonesia vehicle license plates. The algorithm includes grayscale, binary, erosion, dilation and convolution processes, as well as the process of smearing, location determination and character segmentation before the ELM algorithm is applied. The algorithm includes one crucial and rarely performed technique for extraction of vehicle license plates, namely Smearing Algorithms. In the experimental results, ELM is compared with the template matching method. The obtained outcome of the average accuracy of both methods has the same value of $70.3175 \%$.
\end{abstract}

Keyword: Idiopathic Thrombocytopenic Purpura, Expert System, KNN, Certainty Factor

\section{Introduction}

Vehicle license plate recognition system has always been an interesting and challenging research material to do. Until the last few years, there are still a lot of publication papers both from the results of seminars or conferences and international journals that discuss this research. Among them can be mentioned include the following: [1] - [6].

Many related applications and research that utilize the results of the detection and recognition of vehicle license plates including automatic toll services, intelligent transportation services, smart parking system, intelligent traffic management, driving safety systems, traffic accident prevention, automatic vehicle guidance systems, traffic violation monitoring crossing, tracking and identification of vehicle license plates that are considered suspicious based on camera readings installed at each highway crossing, and others.

Vehicle license plate detection and recognition systems in many studies are known as Automatic License Plate Recognition (ALPR) [7], Automatic Vehicle Identification (AVI) [8], Car Plate Recognition (CPR) or Car License plate Recognition (CNPR) [9], Automatic License plate Recognition (ANPR) [10], and Optical Character Recognition (OCR) for cars [11].

The general approach used for the detection and recognition of vehicle license plates usually uses visionbased techniques from images or videos. However, due to the many problems of vehicle license plates including color, size, orientation, shape and pose of vehicle license plates, making the development of detection systems and recognition of vehicle license plates that are automatic, robust and effective becomes a challenge in itself. Not to mention the diversity of vehicle license plates in various countries makes the use of the method of detecting and recognizing vehicle license plates not be used universally. Generally, each country has its own license plates, this needs specific and unique methods of detecting and recognizing license plates.

Several problems of the types of vehicle license plates or the environment that are a challenge in the detection and recognition of vehicle license plates, among others can be mentioned as follows [12]:

\subsection{Vehicle license plate variations}

a) Location (position): the vehicle license plates are in a different location (position) for each vehicle;

b) Quantity: a picture of a vehicle may not be present or many (more than one) plates;

c) Size: vehicle license plates may vary in size depending on the distance taken by the camera and zoom factor (zoom factor);

d) Color: vehicle license plates can be of various characters (letters and numbers) and background colors according to the type of plate or shooting equipment.

\footnotetext{
* Corresponding author : basukirahmat.if@upnjatim.ac.id
} 
e) Letters: license plates in one country and another may differ in the use of different types of letters and languages;

f) Standard and nonstandard: each country has a vehicle license plate standard rule; however, many vehicles are found using non-standard vehicle license plates;

g) Occlusion: vehicle license plates can be obscured by dirt;

h) Tendency: vehicle license plates can be tilted;

i) Others: addition of characters, vehicle license plates are given frames and screws.

\subsection{Environmental Variations}

a) llumination: input images may have different types of lighting, mainly due to the lighting environment and vehicle lights;

b) Background: the background image may have a pattern similar to the vehicle license plate, such as the number printed on the vehicle, the bumper with vertical patterns, and the textured floor.

The things above make research into the detection and introduction of vehicle license plates as never finished and there are always gaps to continue to be developed for future progress. On the other hand, the development of smart computing or soft computing provides opportunities for the emergence or discovery of new methods in the development of algorithms and mathematical modeling to solve the problem of the introduction of the vehicle license plates.

One soft computing method that can be used for vehicle license plate character recognition is the Extreme Learning Machine (ELM). ELM is a new learning method from Artificial Neural Networks (ANN). ANN itself has been widely used as a learning machine, especially for the purposes of pattern recognition [13] - [17]. The ELM method was first introduced by Huang [18]. ELM is a feedforward ANN with a single hidden layer or commonly referred to as a Single Hidden Layer Feedforward Neural Networks (SLFNs).

ELM learning methods are made to overcome the weaknesses of feedforward neural networks, especially in terms of learning speed. Two reasons why Feedforward ANN has a low learning speed, namely [18]:

1. Use a slow gradient-based learning algorithm to conduct training.

2. All parameters on the network are determined iteratively using the learning method.

In learning by using a conventional gradient-based learning algorithm such as backpropagation (BP) and Lavenberg Marquardt (LM) variants, all parameters in feedforward ANN must be determined manually. The parameters in question are the input weight and hidden bias. These parameters are also interconnected between one layer to another, so it requires a long learning speed and is often trapped in local minima [19]. Whereas in ELM parameters such as input weight and hidden bias are chosen randomly, so that ELM has a fast-learning speed and is able to produce good generalization performance. Figure 1 is the structure of ELM [20].

The ELM method has a mathematical model that is different from feedforward ANN. The mathematical model of ELM is simpler and more effective. With just as much $\mathrm{N}$ training data $\left(v_{i}, t_{i}\right)$, whereas $v_{i}=\left[v_{i 1}, v_{i 2}, \ldots, v_{i n}\right]^{T} \in R^{n}$ network input vectors, and $t_{i}=\left[t_{i 1}, t_{i 2}, \ldots, t_{i m}\right]^{T} \in R^{m}$ as desired targets, standard SLFNs with as many hidden neurons $\widetilde{N}$ and activation functions $\phi(v)$ can be modeled as in (1) [21].

$$
\sum_{i=1}^{\bar{N}} \beta_{i} \phi\left(w_{i} \cdot v_{j}+b_{i}\right)=\text { Out }_{j}, \quad j=1, \ldots, N
$$

where $w_{i}=\left[w_{i 1}, w_{i 2}, \ldots, w_{i n}\right]^{T}$ is the interconnection weight vector between as many hidden neurons as $i$ and input neurons, $\beta_{i}=\left[\beta_{i 1}, \beta_{i 2}, \ldots, \beta_{i m}\right]^{T}$ is the interconnection weight vector between as many as $\mathrm{i}$ hidden neurons and output neurons, and $b_{i}$ is the threshold value of as many hidden neurons as i. While $w_{i} \cdot v_{j}$ stating the product matrix (inner product) between $w_{i}$ and ${ }^{v_{j}}$. SLFNs network output is chosen to be near linear.

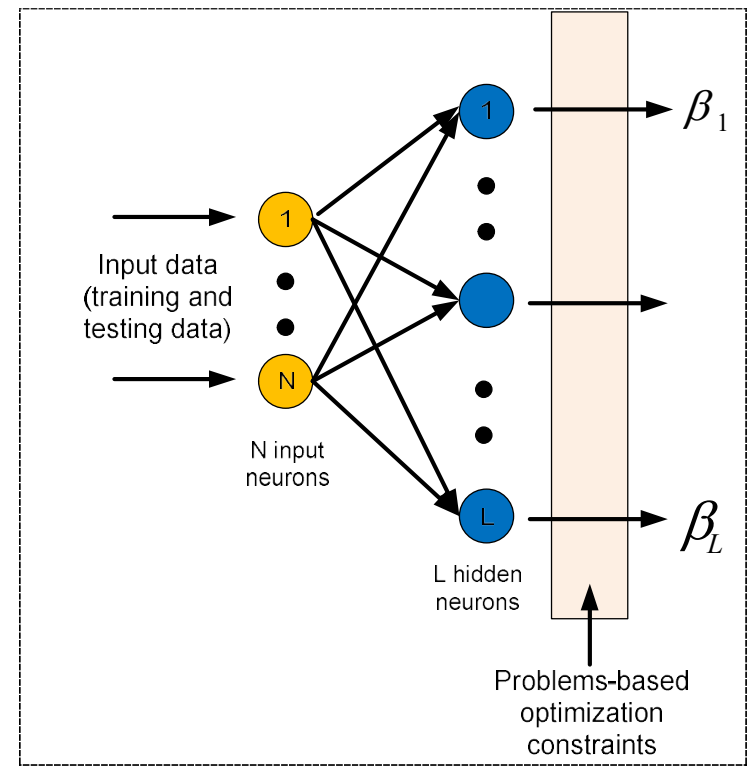

Fig.1. ELM structure.

In standard SLFNs with $\breve{N}_{\text {hidden neurons and the }}$ activation function $\phi(v)$ of several $\mathrm{N}$ training data samples, the goal is approaching zero error. In other words, the network output minus the target is equal to zero, or $\sum_{j=1}^{N}\left\|O u t_{j}-t_{j}\right\|=0$. If the value $\beta_{i}, w_{i}$ and $b_{i}$ are present, then (1) can be expressed in (2) [21]. 


$$
\sum_{i=1}^{\bar{N}} \beta_{i} \phi\left(w_{i} \cdot v_{j}+b_{i}\right)=t_{j}, \quad j=1, \ldots, N
$$

Equation (2) can be written as simply as in (3) [21]

$$
H \beta=T
$$

where

$$
\begin{aligned}
& H\left(w_{1}, \ldots, w_{\bar{N}}, b_{1}, \ldots, b_{\bar{N}}, v_{1}, \ldots, v_{N}\right) \\
& =\left[\begin{array}{ccc}
\phi\left(w_{1} \cdot v_{1}+b_{1}\right) & \cdots & \phi\left(w_{\tilde{N}} \cdot v_{1}+b_{\tilde{N}}\right) \\
\vdots & \cdots & \vdots \\
\phi\left(w_{1} \cdot v_{N}+b_{1}\right) & \cdots & \phi\left(w_{\tilde{N}} \cdot v_{N}+b_{\tilde{N}}\right)
\end{array}\right] N x \breve{N}
\end{aligned}
$$

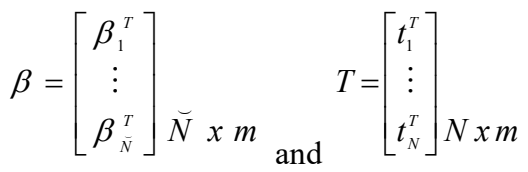

$\mathrm{H}$ is the output matrix of the hidden layer of SLFNs or ELM networks. Column number i of $\mathrm{H}$ is the output vector of number $\mathrm{i}$ hidden neurons due to input $v_{1}, v_{2}, \ldots, v_{N}$.

Broadly speaking, the stages of the ELM method are divided into three stages, namely preprocessing, training, and testing. In recognizing vehicle license plate characters, ELM is used to recognize letters and numbers contained on vehicle license plates. The training process is carried out after the segmentation process of the license plate of the vehicle. Likewise, testing is also applied to the license plates of other vehicles outside the training.

Other methods of character recognition that have been carried out include using ANN [14], deep neural network [22]. As for the recognition of vehicle license plate characters themselves that have been developed include using Artificial Neural Network Classifier methods [23], Supervised K-means and Support Vector Machines [24], Extremal Regions and Restricted Boltzmann Machines [25], and Deep Convolutional Neural Networks [26], [27].

\section{Methodology}

The proposed recognition of vehicle license plate characters by ELM is shown in Figure 2. The recognition system includes grayscale, binarization, erosion, dilation and convolution processes, smearing and location determination, and character segmentation. Afterward, the ELM algorithm is applied. Grayscale, binarization, erosion, dilation, and convolution are commonly implemented for pre-processing part. In the algorithm, there is one important and seldom implemented technique for extraction of vehicle license plates called the Smearing Algorithm.

The smearing Algorithm is one method that can be used for the extraction of vehicle license plates [28]. In this paper, the Smearing Algorithm is intended for the extraction of text areas on the vehicle license plate image. With this Smearing Algorithm, images are processed along with vertical and horizontal rotation. The method is performed by processing the image into horizontal and vertical lines to extract the area of only certain parts and translate the area to white and black from the original color.

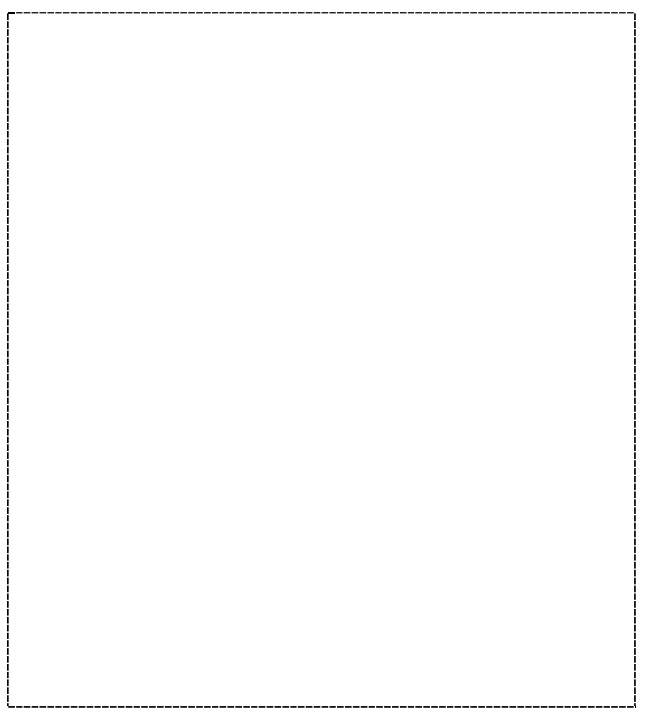

Fig.2. Flow chart proposed methodology.

The smearing process identifies and counts white pixels, and then smears them with white. This result was identified as a license plate region. Whereas besides that if the pixels are not included in the range, the area will be smeared with black.

In the extraction of a vehicle's license plate, after the image is cropped and extracted only shows the area of the license plate region, the smearing process is then used to scan every single character horizontally and vertically. Each character is identified by its actual location and separated from the merge with other characters by reading white pixels on each character. Smearing Algorithms to extract vehicle license plates are shown in Figure 3.

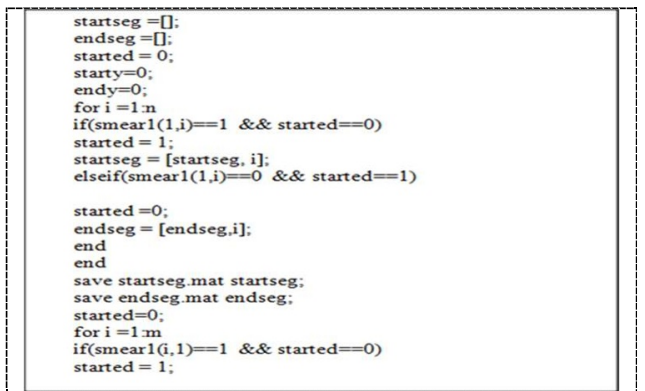

Fig.3. Smearing Algorithm.

From the Smearing Algorithm code in Figure 3, scanning all characters one by one starts from the first character in the horizontal line. The first character that is in the white pixel (0), will be counted and scanned from the first point of the white pixel and stops at the end of the white pixel. Initial $=0$; Endy $=0$; Until black pixels are found with the if code ( $\operatorname{smear} 1(i, 1)==1 \& \&$ started $==0$ ). In the same way for black pixels (1). Initial $=1$; Endy $=1$; Until the white pixel area is found again with the if code $(\operatorname{smear} 0(i, 1)==1 \& \&$ start $==1)$. After each character is scanned by this function, the character will be saved. 


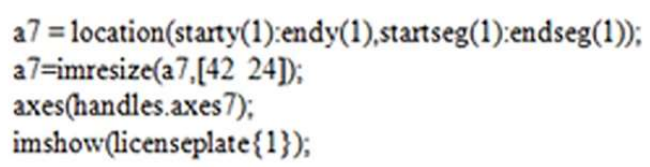

Fig.4. Location determination function.

The Smearing Algorithm to produce a vehicle license plate extraction output must still be added to the location determination function as shown in Figure 4. Explanation of the first line code from Figure 4, where a7 = location (starty (1): endy (1), starteg (1): endseg (1)). This function is basically to save and capture every character from the previous execution. The characters are then resized to $42 \times 24$, code a $7=$ imresize (a7, [42 24]). The size must be adjusted so that the comparison process is easier. Next, the final code is axes (handles. axes7) and imshow (licenseplate $\{1\}$ ), to display the results of the Smearing Algorithm process and its location determination. An example of the output of the Smearing Algorithm is shown in Figure 5.

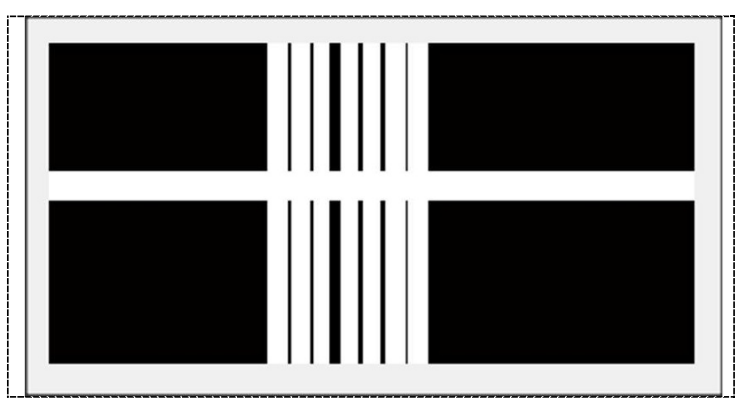

Fig.5. Example of Smearing Algorithm output.

Furthermore, from the output of the Smearing Algorithm process, a character segmentation process is carried out. Subsequently, the ELM method is applied to recognize the license plate characters.

To determine the accuracy of character recognition for each vehicle license plate, it is done by counting the number of characters that have been identified divided by the total number of characters found on the vehicle license plate, multiplied by $100 \%$. With $\mathrm{N}$ the number of characters on the license plate of the vehicle being tested, the calculation of accuracy is defined as in (6).

$$
\operatorname{Accuracy}(\%)=\frac{\sum_{i=1}^{N} \text { Recognized characters }(i)}{N} \times 100 \%
$$

\section{Results}

After implementing the proposed ELM method, the next step is to conduct a trial run. A trial is conducted to obtain the performance produced by the ELM method for the recognition of vehicle license plates. Data input on this trial are in the form of a front view vehicle image.

An output in the form of the results of the segmentation process is obtained by applying grayscale, binarization, erosion, dilation and convolution processes, as well as smearing and location determination along with character segmentation. This character from the segmentation process will then be recognized using the ELM algorithm. The training data used in this study is a picture of numbers and letters which are vehicle license plate characters, namely the numbers $0-9$ and the letter $A$ to $Z$. The results of the ELM training process is shown in Table 1.

Table 1. ELM Training Process Results.

\begin{tabular}{cccc}
\hline \multirow{2}{*}{$\begin{array}{c}\text { Character } \\
\text { Training } \\
\text { Data }\end{array}$} & $\begin{array}{c}\text { Number } \\
\text { of } \\
\text { hidden } \\
\text { neurons }\end{array}$ & \multicolumn{2}{c}{$\begin{array}{c}\text { Results of the Training } \\
\text { Process }\end{array}$} \\
\cline { 3 - 4 } & RMSE & $\begin{array}{c}\text { Processing } \\
\text { Time } \\
\text { (seconds) }\end{array}$ \\
\hline $\begin{array}{c}0-9 \text { dan A- } \\
\text { Z }\end{array}$ & 1000 & $5.6459 \mathrm{e}-14$ & 31.5781 \\
\hline
\end{tabular}

The display of the vehicle license plate character recognition program with ELM is shown in Figure 6. An example of the results of the recognition of a vehicle license plate character by ELM is shown in Figure 7. In full, the results of testing the recognition of vehicle license plates using ELM compared to the Matching Template are presented in Table 2.

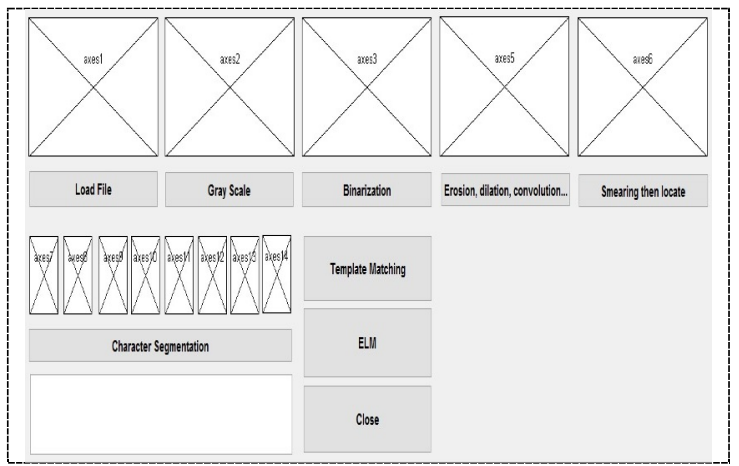

Fig. 6. Display the vehicle license plate character recognition program using ELM.

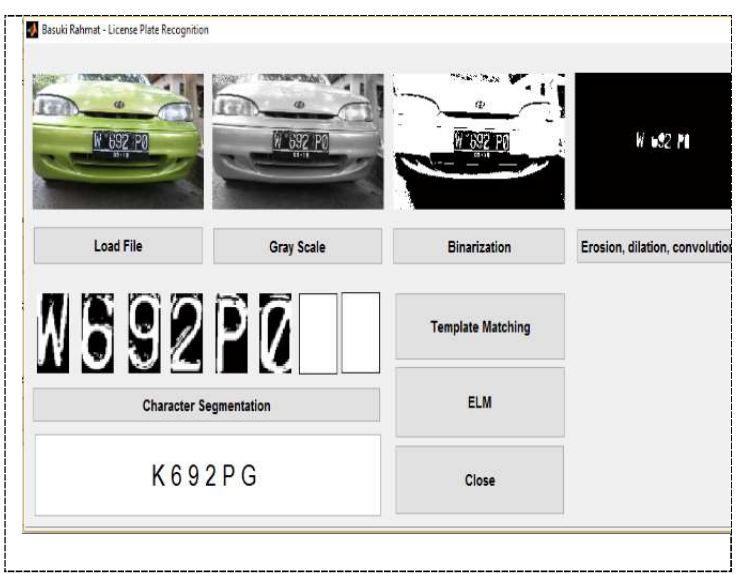

Fig.7. Example of the results of the vehicle license plate character recognition using ELM. 
Table 2. Results of Vehicle License Plate Characters Recognition Using ELM

\begin{tabular}{|c|c|c|c|c|c|c|c|c|c|c|}
\hline \multirow[b]{2}{*}{ Num } & \multirow{2}{*}{$\begin{array}{c}\text { Image } \\
\text { JPG }\end{array}$} & \multirow[b]{2}{*}{ Grayscale } & \multirow[b]{2}{*}{ Binarization } & \multirow{2}{*}{$\begin{array}{l}\text { Erosion, } \\
\text { Dilation, } \\
\text { Convolution }\end{array}$} & \multirow{2}{*}{$\begin{array}{c}\text { Smearing } \\
\text { and } \\
\text { location } \\
\text { Determination }\end{array}$} & \multirow{2}{*}{$\begin{array}{c}\text { Segmen- } \\
\text { tation } \\
\text { Results }\end{array}$} & \multicolumn{2}{|c|}{$\begin{array}{l}\text { Template } \\
\text { Matching }\end{array}$} & \multicolumn{2}{|c|}{ ELM } \\
\hline & & & & & & & Results & $\begin{array}{l}\text { Accur } \\
\text { acy } \\
(\%)\end{array}$ & Results & $\begin{array}{c}\text { Accur } \\
\text { acy } \\
(\%) \\
\end{array}$ \\
\hline 1 & & & & $1 \operatorname{sen}$ & & 10030! & K503СL & 71.4286 & K503टL & 71.4286 \\
\hline 2 & 2. & & & $\therefore \approx$ & & 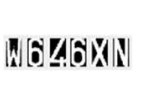 & $\begin{array}{c}W 545 X \\
N\end{array}$ & $\begin{array}{c}66.666 \\
7\end{array}$ & $\begin{array}{c}W 545 X \\
N\end{array}$ & $\begin{array}{c}66.666 \\
7\end{array}$ \\
\hline 3 & & & & $4 \sin 4$ & & Livgs9!? & $\begin{array}{c}\operatorname{LT} 9698 \\
4\end{array}$ & 57.1429 & $\begin{array}{c}\text { LTS690 } \\
\text { L }\end{array}$ & 71.4286 \\
\hline 4 & $\frac{6}{6.1346006}$ & 8 & & . $1346 \mathrm{E}$ & & DUSEGaZ & $\begin{array}{c}\mathrm{LT} 346 \mathrm{E} \\
\mathrm{L}\end{array}$ & 71.4286 & $\begin{array}{c}\text { L7346E } \\
\text { L }\end{array}$ & 71.4286 \\
\hline 5 & 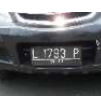 & & & L 1939 & & П & LY793P & $\begin{array}{c}83.333 \\
3\end{array}$ & LX793P & $\begin{array}{c}83.333 \\
3\end{array}$ \\
\hline 6 & & & & $\mathrm{w} 1203 \mathrm{x}$ & & MilDes3) & $\begin{array}{c}\text { W7233X } \\
8\end{array}$ & 71.4286 & $\begin{array}{c}\text { W7233X } \\
\text { W }\end{array}$ & 71.4286 \\
\hline 7 & & & & L ISA tis & & 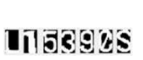 & LT5390S & 71.4286 & LT5390L & 71.4286 \\
\hline 8 & & & & L ssg it & & I99.9[1] & L999A4P & $\begin{array}{c}66.666 \\
7\end{array}$ & L99954L & $\begin{array}{c}66.666 \\
7\end{array}$ \\
\hline 9 & & & & $4467 \mathrm{~s}$ & & INZ67s & LI4B7S & 71.4286 & L14B7S & 71.4286 \\
\hline 10 & & & & psosm & & DQ367L & $\begin{array}{c}04367 \mathrm{~N} \\
\mathrm{~N}\end{array}$ & 71.4286 & 04367N0 & 57.1429 \\
\hline 11 & & & & $154 \mathrm{si}$ & & 15UISV & L5T8SV & 71.4286 & L5T8SV & 71.4286 \\
\hline 12 & 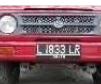 & $\sqrt{0 .}$ & & L 1334 & & $1 \mathrm{gsil}$ & L833LR & 85.7143 & LOBBLLR & 71.4286 \\
\hline 13 & 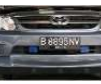 & 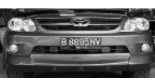 & & & & В & $\begin{array}{c}\text { BM95N } \\
V\end{array}$ & 71.4286 & $\begin{array}{c}\mathrm{BM} 95 \mathrm{~N} \\
\mathrm{~V}\end{array}$ & 71.4286 \\
\hline 14 & & 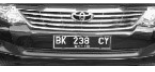 & & & & L'L23:Ga & $\begin{array}{c}\text { TK239С } \\
7\end{array}$ & 57.1429 & ВК239СВ & 71.4286 \\
\hline 15 & $\frac{1}{102 x+10}$ & $\frac{2}{20}$ & 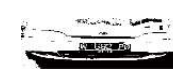 & $n+\infty$ & & NG9280 & $\begin{array}{c}\text { KLIQP } \\
0\end{array}$ & $\begin{array}{c}66.666 \\
7\end{array}$ & $\begin{array}{c}\text { KUIY2P } \\
0\end{array}$ & $\begin{array}{c}66.666 \\
7\end{array}$ \\
\hline & & & Average Acc & acy Results & & & & 70.3175 & & 70.3175 \\
\hline
\end{tabular}


From 15 times of the license plate character recognition test of the vehicle, the following results were obtained. ELM compared to Template Matching, 11 times the same results were obtained, 2 times better, namely in the 3 rd and 14th tests, and 2 times worse, namely in the 10th and 12th tests. In the final result, the average accuracy of the two methods is the same, which is $70.3175 \%$. Although the results are the same, the ELM algorithm still has advantages, namely in the form of an ELM network architecture with the weights of the results of the training. The results of this training process indicated that ELM can be used as a learning machine with the ability to remember learned patterns that are not owned by the Matching Template.

\section{Conclusion}

The recognition of vehicle license plate characters by means of ELM was considered in this paper. In addition, the considered recognition algorithm utilized the Smearing Algorithm as the vehicle license plate extraction. Simulation results were conducted to acquire the performances of the proposed recognition method. Comparison results with the template matching method confirmed that the two methods produce the same performance on average accuracy.

\section{References}

[1] D. C. Luvizon, B. T. Nassu, and R. Minetto, "A Video-Based System for Vehicle Speed Measurement in Urban Roadways," IEEE Trans. Intell. Transp. Syst., vol. 18, no. 6, pp. 13931404, Jun. (2017).

[2] A. J. Fernández-Ares, A. M. Mora, S. M. Odeh, P. García-Sánchez, and M. G. Arenas, "Wireless monitoring and tracking system for vehicles: A study case in an urban scenario," Simul. Model. Pract. Theory, vol. 73, pp. 22-42, (2017).

[3] K. (Kate) Hyun, A. Tok, and S. G. Ritchie, "Long distance truck tracking from advanced point detectors using a selective weighted Bayesian model," Transp. Res. Part C Emerg. Technol., vol. (82), pp. 24-42, (2017).

[4] S. Khakpour, R. W. Pazzi, and K. El-Khatib, "Using clustering for target tracking in vehicular ad hoc networks," Veh. Commun., vol. 9, pp. 8396, (2017).

[5] M. K. Saini and S. Saini, "Multiwavelet Transform Based License plate Detection," J. Vis. Commun. Image Represent., p. , (2017).

[6] M. Anandhalli and V. P. Baligar, "A novel approach in real-time vehicle detection and tracking using Raspberry Pi," Alexandria Eng. J., (2017).

[7] R. M. Khoshki and S. Ganesan, "Improved Automatic License Plate Recognition (ALPR) system based on single pass Connected Component Labeling (CCL) and reign property function," (2015), pp. 426-431.

[8] Y. Chang, Z. Su, and L. Qian-Yu, "A New Traffic Incident Detection Method Under Low-
Volume Condition Based On Automatic Vehicle Identification," in 2012 9th International Conference on Fuzzy Systems and Knowledge Discovery, (2012), pp. 2853-2859.

[9] J. A. Khan and M. A. Shah, "Car License plate Recognition (CNPR) system using multiple template matching," in 2016 22nd International Conference on Automation and Computing (ICAC), (2016), pp. 290-295.

[10] M. Zhu, C. Liu, J. Wang, X. Wang, and Y. Han, "A Service-Friendly Approach to Discover Traveling Companions Based on ANPR Data Stream," in 2016 IEEE International Conference on Services Computing (SCC), (2016), pp. 171178.

[11] A. Jain and J. Sharma, "Classification and interpretation of characters in multi-application OCR system," in 2014 International Conference on Data Mining and Intelligent Computing (ICDMIC), (2014), pp. 1-6.

[12] S. Du, M. Ibrahim, M. Shehata, and W. Badawy, "Automatic License Plate Recognition (ALPR): A State-of-the-Art Review," Circuits Syst. Video Technol. IEEE Trans., vol. 23, no. 2, pp. 311325, Feb. (2013).

[13] C. M. Costa, I. S. Silva, R. D. de Sousa, R. A. Hortegal, and C. D. M. Regis, "The association between reconstructed phase space and Artificial Neural Networks for vectorcardiographic recognition of myocardial infarction," $J$. Electrocardiol., vol. 51, no. 3, pp. 443-449, (2018).

[14] T. Akter and S. Desai, "Developing a predictive model for nanoimprint lithography using artificial neural networks," Mater. Des., vol. 160, pp. 836-848, (2018).

[15] I. Kucukoglu, H. Atici-Ulusu, T. Gunduz, and O. Tokcalar, "Application of the artificial neural network method to detect defective assembling processes by using a wearable technology," $J$. Manuf. Syst., vol. 49, pp. 163-171, (2018).

[16] P. Sudha, D. Ramyachitra, and P. Manikandan, "Enhanced Artificial Neural Network for Protein Fold Recognition and Structural Class Prediction," Gene Reports, vol. 12, pp. 261-275, (2018).

[17] S. van Cranenburgh and A. Alwosheel, "An artificial neural network based approach to investigate travellers' decision rules," Transp. Res. Part C Emerg. Technol., vol. 98, pp. 152166, (2019).

[18] G.-B. Huang, Q.-Y. Zhu, and C.-K. Siew, "Extreme learning machine: theory and applications," Neurocomputing, vol. 70, no. 1, pp. 489-501, (2006).

[19] G.-B. Huang, M.-B. Li, L. Chen, and C.-K. Siew, "Incremental extreme learning machine with fully complex hidden nodes," Neurocomputing, vol. 71, no. 4, pp. 576-583, (2008).

[20] K. Demertzis and L. Iliadis, "Bio-inspired hybrid intelligent method for detecting android malware," in Knowledge, Information and 
Creativity Support Systems, Springer, (2016), pp. 289-304.

[21] G.-B. Huang, Q.-Y. Zhu, and C.-K. Siew, "Extreme learning machine: a new learning scheme of feedforward neural networks," in Neural Networks, 2004. Proceedings. 2004 IEEE International Joint Conference on, (2004), vol. 2, pp. 985-990.

[22] D. Lin, F. Lin, Y. Lv, F. Cai, and D. Cao, "Chinese Character CAPTCHA Recognition and performance estimation via deep neural network," Neurocomputing, vol. 288, pp. 11-19, (2018).

[23] H. M. Alyahya, M. K. Alharthi, A. M. Alattas, and V. Thayananthan, "Saudi License Plate Recognition System Using Artificial Neural Network Classifier," in 2017 International Conference on Computer and Applications (ICCA), (2017), pp. 220-226.

[24] W. C. Liu and C. H. Lin, "A hierarchical license plate recognition system using supervised Kmeans and Support Vector Machine," in 2017 International Conference on Applied System Innovation (ICASI), (2017), pp. 1622-1625.

[25] C. Gou, K. Wang, Y. Yao, and Z. Li, "Vehicle License Plate Recognition Based on Extremal Regions and Restricted Boltzmann Machines," IEEE Trans. Intell. Transp. Syst., vol. 17, no. 4, pp. 1096-1107, Apr. (2016).

[26] S. M. Silva and C. R. Jung, "Real-Time Brazilian License Plate Detection and Recognition Using Deep Convolutional Neural Networks," in 2017 30th SIBGRAPI Conference on Graphics, Patterns and Images (SIBGRAPI), (2017), pp. 55-62.

[27] D. N. T. How and K. S. M. Sahari, "Character recognition of Malaysian vehicle license plate with deep convolutional neural networks," in 2016 IEEE International Symposium on Robotics and Intelligent Sensors (IRIS), (2016), pp. 1-5.

[28] L. Teo and F. Wong, "Smearing algorithm for vehicle parking management system," in Proceedings of the 2nd Seminar on Engineering and Information Technololgy, (2009), pp. 331337. 